\title{
Semiconductor Microspheres in Optoelectronics
}

\author{
Ali Serpengüzel*, Yigit Ozan Yilmaz, Abdullah Demir, Onur Akatlar, and Adnan Kurt \\ Koç University, Microphotonics Research Laboratory, Physics Department, \\ Rumeli Feneri Yolu, Sariyer, Istanbul 34450 Turkey
}

\begin{abstract}
Semiconductor microspheres coupled to optical fibers are used for optical channel dropping in the IR communication wavelenghts of 800 to $1500 \mathrm{~nm}$. The observed morphology dependent resonances have quality factors of 100000 . The measured quality factors are limited by the sensitivity of the experimental setup. These optical resonances provide the necessary narrow linewidths, that are needed for high resolution optical communication applications. In addition to optical communication, detection, and switching applications of this optoelectronic system is studied experimentally and theoretically. The microsphere, optical fiber system shows promise as a building block for optoelectronic integration.
\end{abstract}

Keywords: Channel dropping filter, microsphere resonator, morphology-dependent resonance, whispering gallery mode.

\section{INTRODUCTION}

Microspheres are uniquely applicable to compact optoelectronic devices for wavelength division multiplexing (WDM) [1]. In optical communication, WDM is important for increasing the bandwidth of the current fiber optic networks. In WDM, the final optical to electronic conversion needs an all-optical packetswitching layer, which consists of all-optical gates, interferometers, semiconductor optical amplifiers, resonant cavity enhanced photodetectors, optical random access memory elements, and channel dropping filters. In these planar lightwave circuits, dielectric $\mu$-spheres with their MDR's, can be used as compact channel dropping filters. In such filters, a microsphere coupled to two optical fibers constitutes an add-drop filter. Optical signals are introduced through one of the optical fibers leading into the filter (add port). Signals at the resonant wavelengths of the filter are transferred to the other optical fiber (drop port) at the other side of the filter. Optical signals at nonresonant wavelengths pass through the filter along the input optical fiber (through port). Fiber optic add-drop filters based on a silica microsphere system on a taperresonator-taper coupler and channel-dropping filter using a microsphere and integrated waveguides have been developed [2]. Si-SiO2 microring resonator optical channel dropping filter was realized with $\mathrm{Q}$ values of 250 in $5-\mu \mathrm{m}$ radius [3].

\footnotetext{
* Correspondence should be addressed to Ali Serpengüzel, aserpenguzel@ku.edu.tr; phone: + 90 (212) 338-1312; fax: + 90 (212) 338-1547, http://home.ku.edu.tr/ aserpenguzel, http://microphotonics.ku.edu.tr, Koç University, Microphotonics Research Laboratory, Physics Department, Rumeli Feneri Yolu, Sariyer, Istanbul 34450 Turkey. The authors are with Koç University, Microphotonics Research Laboratory, Department of Physics, Rumeli Feneri Yolu, Sariyer, Istanbul 34450 Turkey.
} 


\section{MORPHOLOGY DEPENDENT RESONANCES}

A physical interpretation of MDRs is based on propagation of rays around the inside surface of the microsphere, confined by an almost total internal reflection (TIR) [4]. The rays approach the internal surface at an angle beyond the critical angle and are totally internally reflected each time. After propagating around the microsphere, the rays return to their respective entrance points exactly in phase and then follow the same path all over again without being attenuated by destructive interference [5]. A MDR is specified by a mode order $l$ (the number of radial intensity maxima) and mode number $n$ (the number of maxima between $0^{\circ}$ and $180^{\circ}$ degrees in the angular distribution of the energy of the mode). MDRs satisfy resonance conditions for specific values of the size parameter $x=2 \pi a / \lambda$, where $a$ is radius of the sphere and $\lambda$ is the vacuum wavelength of light [6].

The MDR's of a $\mu$-sphere are analyzed by the localization principle [7] and the generalized Lorenz-Mie theory (GLMT) [8][9][10]. Each MDR is characterized by a mode number (n) and a mode order (l). The angular mode number (n) gives the number of maxima between $0^{\circ}$ and $180^{\circ}$ degrees in the polar angular distribution of the energy of the MDR. The radial mode order (l) indicates the number of maxima in the internal electric field distribution in the radial direction. Each MDR of the $\mu$-sphere also has an azimuthal angular dependence from $0^{\circ}$ to $360^{\circ}$, which is labeled with an azimuthal mode number. However, for spheres, MDR's differing only in azimuthal mode number have identical resonance frequencies [11].

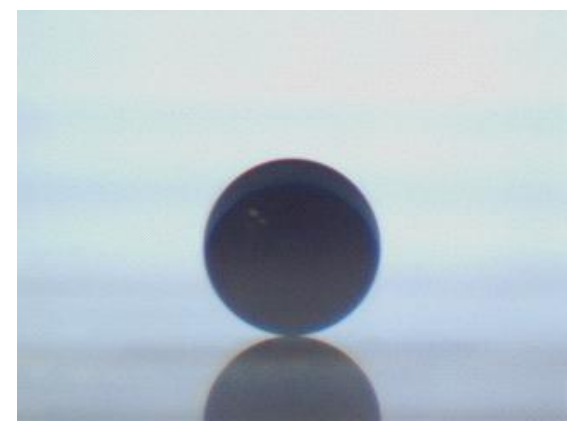

Fig. 1. The picture of the silicon microsphere.

The greatest impediment to the use of $\mu$-sphere resonators in practical devices has been the difficulty of efficiently coupling light into and out of the $\mu$-spheres. Light can be coupled into the microsphere using different types of coupling devices: side-polished optical fibers [12], prisms [13] and tapered optical fibers [14]. The principle of these devices is based on providing efficient energy transfer to the resonant circular TIR guided wave in the $\mu$-sphere resonator (representing the MDR mode) through the evanescent field. Efficient coupling can be expected if two main conditions are satisfied: phase synchronism and significant overlap of the two waves modeling the MDR mode and the coupler mode. The coupling is based on the fact that the modal field of the guided mode extends beyond the core-cladding interface [15]. A silicon microsphere coupled to polished half-block used in the experiments is shown in Fig. 1.

\section{SILICON MICROSPHERES}

Silicon has been the material of choice for the electronics industry for more than half-a-century. It is a relatively inexpensive, plentiful, and well understood material for producing electronic devices [16]. Additionally, the need for low cost photonic devices has stimulated a significant amount of research in 
silicon photonics [17]. Although silicon photonics is less well developed as compared to III-V technologies; it is poised to make a serious impact on the telecommunications industry, as well as in many other photonic applications. Recent studies show that the silicon will be widely used, as in electronics, in the photonics industry [18]. Silicon microspheres with their MDRs will be an additional step for the use of silicon in optical communication applications. The MDR peaks in the elastic scattering spectrum and associated dips in the transmission spectrum are experimentally observed by coupling the light from an optical fiber half coupler (OFHC) to the MDRs of a silicon $\mu$-sphere placed on the OFHC surface. Silicon $\mu$-spheres with their high Q MDRs of $10^{5}$ can be used as active channel-dropping filters. The Q-factor of a $\mathrm{MDR},(\mathrm{Q}=\lambda / \delta \lambda)$, gives information about the lifetime and linewidth of the resonance [19].

\section{EXPERIMENTAL SETUP}

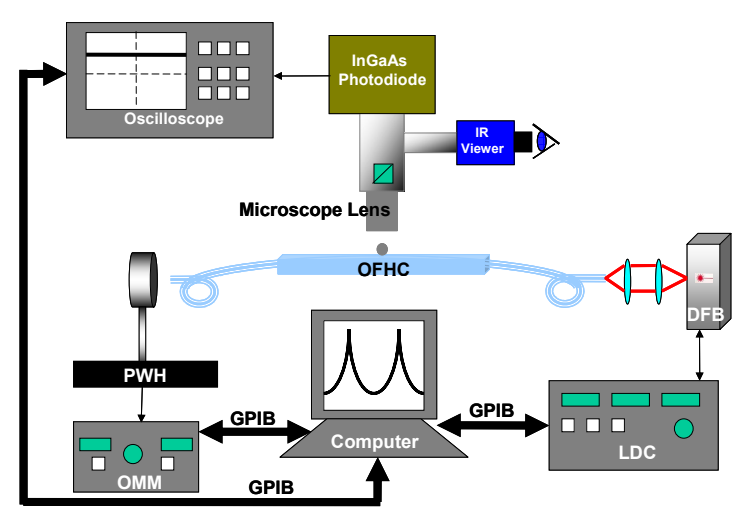

Fig. 2. The schematic of the experimental setup of the optical channel dropping.

The schematic of the experimental setup is shown in Fig. 2. A tunable distributed feedback (DFB) laser is used to excite the MDR's of the silicon microsphere. Wavelength tuning is achieved by tuning the temperature of the DFB laser with a laser diode controller (LDC). Laser light is coupled into the OFHC. The optical fiber used in the OFHC is a standard $1300 \mathrm{~nm}$ single-mode fiber. The silicon $\mu$-sphere used in the experiment with $a=530 \mu \mathrm{m}$ and $n=3.5$ is placed on the interaction region of the OFHC. The scattered light from the microsphere at $90^{\circ}$ is collected by a microscope lens and detected by an InGaAs photodiode. The transmitted power through the optical fiber is detected by an optical multimeter (OMM) with InGaAs power/wave head (PWH). The InGaAs photodiode signal is sent to the digital oscilloscope for signal monitoring and data acquisition. An infrared viewer is used to observe the scattered light from the microsphere. Data acquisition and control are performed with IEEE-488 GPIB interface.

\section{EXPERIMENTAL RESULTS}




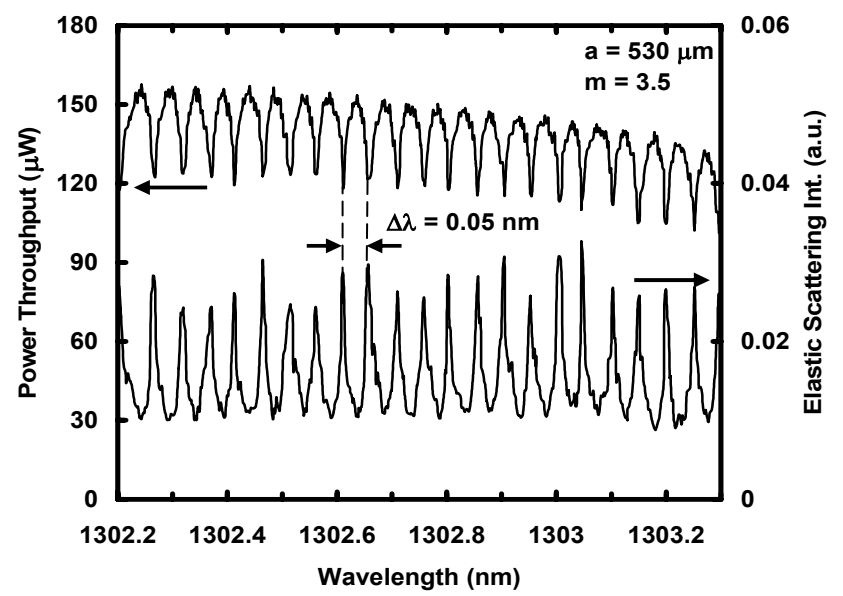

Fig. 3. The elastic scattering intensity and power transmission spectra from the silicon microsphere.

Fig. 3 shows the elastic scattering spectrum at a scattering angle of $90^{\circ}$ from the silicon microsphere and the power transmission spectrum. In the elastic scattering, there is a background due to OFHC surface imperfections. The temperature of the DFB laser is tuned from $18^{\circ} \mathrm{C}$ to $34^{\circ} \mathrm{C}$ at a constant current of 29 $\mathrm{mA}$. The temperature range corresponds to $1302.18 \mathrm{~nm}$ to $1303.30 \mathrm{~nm}$. The dips in the transmission spectrum are due to the coupled MDR wavelengths into the microsphere (which results in incident power losses). The mode spacing $\Delta \lambda$ (i.e. wavelength difference between consecutive mode numbers in the same mode order) is measured to be $0.19 \mathrm{~nm}$, which is in agreement with the Mie scattering theory, where mode spacing is given by $\Delta \lambda=\left(\lambda^{2} \arctan \left(\mathrm{m}^{2}-1\right)^{1 / 2}\right) /\left(2 \pi \mathrm{a}\left(\mathrm{m}^{2}-1\right)^{1 / 2}\right)$ [20]. The linewidths $\delta \lambda$ of the MDRs are measured to be approximately $0.007 \mathrm{~nm}$ which correspond to a Q-factor of $2 \times 10^{5}$. [21]

\section{CONCLUSIONS}

We have demonstrated the excitation of MDR's of silicon microspheres using an optical fiber half coupler (OFHC) and, a temperature tunable distributed feedback (DFB) laser. MDR peaks in the elastic scattering spectra and associated dips in the transmission spectra are observed experimentally. With the proper system design, it would be possible to totally drop the selected MDR's power from the transmission spectrum. The microsphere, optical fiber, and detector system shows promise as a building block for optoelectronic integration. the results showed that silicon microspheres are strong candidates for filtering applications in optical communication systems. Additionally, the silicon microspheres with their high Q MDRs can be integrated into to the all-optical silicon photonic devices. Silicon, being a semiconductor, opens up the way for the realization of active photonic devices.

\section{ACKNOWLEDGMENTS}

We would like to acknowledge the partial support of this research by the Türkiye Bilimsel ve Teknolojik Arastirma Kurumu (The Scientific and Technical Research Council of Turkey-TUBITAK) under Grant TBAG 1952 and in part by the European Office of Aerospace Research and Development (EOARD) under Grants F61775-01-WE062 and FA8655-02-M4086 and the European Commission (EC) Grants No: FP6IST-003887 NEMO and FP6-IST-511616 PHOREMOST.

\section{REFERENCES}


[1] Y.O. Yilmaz, A. Demir, A. Kurt, and A. Serpengüzel, "Optical Channel Dropping with a Silicon Microsphere," IEEE Photon. Technol. Lett. 17, ******* (2005).

[2] M. Cai, G. Hunziker, and K. Vahala, "Fiber optic add-drop device based on a silica microsphere-whispering gallery mode system," IEEE Photon. Techn. Lett., vol. 11, pp. 686-687, June1999.

[3] B. E. Little, J. S. Foresi, G. Steinmeyer, E. R. Thoen, S. T. Chu, H., A. Haus, E. P. Ippen, L. C. Kimerling, and W. Greene, "Ultra-compact $\mathrm{Si}-\mathrm{SiO} 2$ microring resonator optical channel dropping filters," IEEE Photon. Technol. Lett., vol. 10, pp. 549551, Apr. 1998.

[4] P. Barber, and R. K. Chang, Optical Effects Associated with Small Particles, Singapore: World Scientific, 1988.

[5] P. Barber, and S. C. Hill, Light Scattering by Particles: Computational Methods. Singapore: World Scientific, 1990.

[6] M. Pelton, and Y. Yamamato, "Ultralow threshold laser using a single quantum dot and a microsphere cavity," Phys. Rev. A, vol. 59, pp. 2418-2421, Mar. 1999.

[7] H.M. Nussenzveig, "Diffraction Effects in Semiclassical Scattering," Cambridge University Press, 1992.

[8] G. Griffel, S. Arnold, D. Taskent, A. Serpengüzel, J. Connolly, and N. Morris, "Morphology Dependent Resonances of a Microsphere, Optical Fiber System,” Opt. Lett., vol. 21, no. 10, pp. 695-697, 1996.

[9] J. A. Lock and G. Gouesbet, "Rigorous justification of the localized approximation to the beam shape coefficients in generalized Lorenz-Mie theory. I. On-axis beams," J. Opt. Soc. Am. A, vol. 11, no. 9, pp. 2503-2515,1994.

[10] G. Gouesbet and J. A. Lock, "Rigorous justification of the localized approximation to the beam shape coefficients in generalized Lorenz-Mie theory. II. Off-axis beams,” J. Opt. Soc. Am. A, vol.11, no. 9, pp. 2516-2525, 1994.

[11] P. W. Barber and R. K. Chang, Eds., "Optical Effects Associated with Small Particles," World Scientific, Singapore, 1988.

[12] Serpengüzel, S. Arnold, and G. Griffel, "Excitation of Resonances of Microspheres on an Optical Fiber", Optics Letters, Vol. 20, No. 7, pp. 654-656, April 1995.

[13] M. L. Gorodetsky and V. S. Ilchenko, "High- $Q$ optical whispering gallery microresonators: precession approach for spherical mode analysis and emission patterns," Opt. Commun. 113, 133-143 (1994).

[14] J. C. Knight, G. Cheung, F. Jacques, and T. A. Birks, "Phased matched excitation of whispering gallery modes by a fiber taper," Opt. Lett., Vol. 22, pp. 1129-1131, Aug. 1997.

[15] A. Serpengüzel, S. Arnold, G. Griffel, and J. A. Lock, "Enhanced coupling to microsphere resonances with optical fibers," $J$. Opt. Soc. Am. B, vol. 14, pp. 790-795, Apr. 1997.

[16] L. Pavesi, "Will silicon be the photonic material of the next millennium?," J.Phys.: Condensed Matter 15, pp. 1169-1196, 2003.

[17] L. Pavesi and D. J. Lockwood, Silicon Photonics. New York: Springer-Verlag, 2004.

[18] O. Boyraz, B. Jalali, "Demonstration of a silicon Raman laser," Optics Express, vol. 12, pp. 5269-5273, Oct. 2004.

[19] M. L. Gorodetsky, A. A. Savchenkov, and V. S. Ilchenko, "Ultimate Q of optical microsphere resonators," Optics Letters, vol. 21, pp. 453-455, Apr. 1996.

[20] R. Jia, D. Jiang, P. Tan, B. Sun, J. Zhang, and Y. Lin, "Photoluminescence study of CdSexS1-x quantum dots in a glass spherical microcavity", Chin. Phys. Lett., vol. 18, no. 10, pp. 1350-1352, 2001.

[21] A. Demir and A. Serpengüzel, "Silica Microspheres for Biomolecular Detection Applications," IEE Proceedings on Nanobiophotonics, 105-107 (2005).. 